\title{
Influence of Contact Types and Uncertainties in the Computation of Independent Contact Regions
}

\author{
Máximo A. Roa and Raúl Suárez
}

\begin{abstract}
Independent Contact Regions provide robustness in front of finger positioning errors during an object grasping. However, different sources of uncertainty may be present like, for instance, the friction model used in grasp planning, indetermination of the friction coefficients, and errors in the model of the object that affect the positions of the boundary points as well as the direction normal to the object surface. These sources have not been previously considered in the computation of the Independent Contact Regions. This paper discusses how to take into account these factors when computing the Independent Contact Regions for discretized objects, i.e. objects described with a cloud or a mesh of points. The considerations provided allow a more robust result for application in grasp synthesis and regrasp planning.
\end{abstract}

\section{INTRODUCTION}

Planning the actions to grasp an object is a complex task, particularly when a multi-finger grasping device is used. When a precision grasp is desired (i.e. grasping the object by using only the fingertips) the problem usually involves different sub-problems that can be summarized as: determining a proper set of contact points on the object surface, determine the proper hand configuration to reach these contact points, and determine the forces that the fingers must apply at each contact. Then, there is still the hard work of controlling the hand joints in order to execute the grasp. In general, the goal of the grasp is to immobilize the object in front of any potential external disturbance (in this context the own weight of the object can be considered an external disturbance), and when this condition is reached, it is said that either the form or force-closure properties are satisfied [1].

A form-closure grasp means that the position of the contacts on the object surface ensure the object immobility, only forces normal to the object boundary at each contact are enough to balance any external perturbation and therefore the grasp does not depend at all on friction. A force-closure grasp means that the object immobility is ensured by the forces applied by the fingers. If there exists friction, a finger can applied forces at the frictional contact point along different directions, and therefore the required number of contacts to produce a force-closure grasp is smaller. For instance, a minimum of 7 frictionless or 4 frictional contacts are required

This work was partially supported by the Spanish Government through the projects DPI2008-02448 and DPI2010-15446

M.A. Roa is with the Institute of Robotics and Mechatronics, German Aerospace Center (DLR e.V.), Wessling, Germany. E-mail: maximo.roagarzonedlr.de

R. Suárez is with the Institute of Industrial and Control Engineering (IOC), Technical University of Catalonia (UPC), Barcelona, Spain. E-mail: raul.suarez@upc.edu to get, respectively, a form- or force-closure grasp on a general 3D object [2]. Obtaining a form- or force-closure grasp depends on the proper selection of the grasp contact points. Several works were already presented dealing with this problem like, for instance, in 2D polygonal [3] or nonpolygonal objects [4], 3D polyhedral objects [5], objects with smooth curved surfaces [6], or 3D discretized objects [7], [8].

In order to provide robustness to the grasps in front of possible finger positioning errors during a grasp execution with $n$ fingers, the concept of Independent Contact Regions was introduced [9], this is a set ICRS of regions $\mathrm{ICR}_{i}$, $i=1, \ldots, n$, on the object boundary such that a finger contact in each region allows a form/force-closure (FC) grasp independently of the exact position of each finger. Finger positioning errors on the object surface may be due to different causes, like for instance joint control errors or the fact that the fingers do not touch the object simultaneously and the object is slightly moved before completing the grasp. A set of regions ICRS is also useful to extend the range of solutions in the search of a hand configuration to perform a given FC grasp [10].

Several works have been presented determining ICRS for 2D polygonal [11] and non-polygonal objects [12], and for 3D polyhedral [5] or general objects [13]. Previous works of the authors have presented an algorithmic approach to compute ICRS on 3D discrete objects using any number $n \geq 7$ of frictionless contacts or $n \geq 3$ frictional contacts by growing the regions around the contact points of a given FC grasp and ensuring a controlled minimum quality for any grasp produced with finger contacts inside the ICRS [14].

The influence of uncertainties on grasp planning has been addressed few times, despite that some amount of uncertainty is inevitable in the real world, and that uncertainties can lead even to lose the FC condition. Friction and contact position uncertainty have been considered in the force closure analysis [15], where the minimum friction coefficient and the maximum uncertainty in position of the contact points that still guarantee an FC grasp are also computed. Uncertainties on shape and contact locations are considered in the vision-based grasp planning process for 2D objects [16], or taken as sources of error that are corrected during the grasp execution [17]. Other approaches deal with the uncertainties in the object geometry by using compliant actuators [18]. However, the consideration of uncertainty in the determination of ICRS has not been tackled before. This work discusses the influence of the type of contact, the uncertainty in the friction coefficient, and the uncertainty in the actual object boundary due to errors in the model 
of the object (which affects the positions of the contact points as well as the direction normal to the object surface). The adjustments required to compute ICRS considering these uncertainties are also provided. These considerations allow a wider use of ICRS both in robust grasp synthesis and in regrasp planning.

The rest of the paper is organized as follows. Section II provides the required background on frictional grasps, and summarizes the procedure already presented to compute ICRS. Section III presents the influence of the friction coefficient and the type of contact on the computation of ICRS, while Section IV discusses the influence of uncertainties in the location and normal direction at a given contact point. Finally, Section V summarizes the work and discusses some future applications.

\section{BACKGROUND}

This section presents the assumptions and some basic background required for the proposed approach, and a summary of the procedure to compute the ICRS already presented in [14].

\section{A. Object and contact model}

The following assumptions are considered to compute the independent contact regions on an arbitrary $3 \mathrm{D}$ object:

- The object surface is represented by any type of mesh, i.e. a set $\Omega$ of $N$ points plus some neighboring information among them (the number of neighbors has no influence on the proposed approach). $N$ is assumed to be large enough so that if a grasp is FC with a given finger in any two neighboring points, then a grasp with such finger in any intermediate position is also FC.

- The points of the mesh are described by position vectors $\boldsymbol{p}_{i}$ measured with respect to a reference system located in the center of mass $(C M)$ of the object, and each point $\boldsymbol{p}_{i}$ has an associated surface normal direction $\hat{\boldsymbol{n}}_{i}$ pointing toward the interior of the object. The discretization of the object should guarantee that the object normal direction varies monotonously between two neighboring points.

The frictional contact between each finger and the object can be modeled as a punctual contact or as a soft finger contact. For a punctual contact, Coulomb's friction model is used, i.e. to avoid slipping the force $\boldsymbol{f}_{i}$ applied at $\boldsymbol{p}_{\boldsymbol{i}}$ must lie inside the friction cone defined by $\boldsymbol{f}_{i}^{t} \leq \mu \boldsymbol{f}_{i}^{n}$, where $\mu$ is the friction coefficient and $\boldsymbol{f}_{i}^{t}$ and $\boldsymbol{f}_{i}^{n}$ are the tangential and normal components of $\boldsymbol{f}_{i}$, respectively. In the 3-dimensional physical space this model is nonlinear and, to simplify it, the friction cone is linearized using an $m$-side polyhedral convex cone. Thus, using $\hat{\boldsymbol{n}}_{i j}$ to represent the unitary vector along the $j$-th edge of the convex cone at the $i$-th contact, a grasping force is given by

$$
\boldsymbol{f}_{i}=\sum_{j=1}^{m} \alpha_{i j} \hat{\boldsymbol{n}}_{i j}, \quad \alpha_{i j} \geq 0
$$

The force $\boldsymbol{f}_{i}$ applied on the object at $\boldsymbol{p}_{i}$ generates a torque $\boldsymbol{\tau}_{i}=\boldsymbol{p}_{i} \times \boldsymbol{f}_{i}$ with respect to $C M . \boldsymbol{f}_{i}$ and $\boldsymbol{\tau}_{i}$ are grouped together into a wrench vector given by

$$
\boldsymbol{\omega}_{i}=\left(\begin{array}{c}
\boldsymbol{f}_{i} \\
\boldsymbol{\tau}_{i} / \rho
\end{array}\right)
$$

where $\rho$ is a parameter with units of length used to adjust the metric of the wrench space [14], and without loss of generality it will be considered unitary here to simplify the expressions. The wrench $\boldsymbol{\omega}_{i j}$ generated by a unitary force $\boldsymbol{f}_{i}$ along an edge of the linearized friction cone, i.e. $\boldsymbol{f}_{i}=\hat{\boldsymbol{n}}_{\boldsymbol{i} \boldsymbol{j}}$, is called a primitive wrench. A grasp defined by the set of contact points $C=\left\{\boldsymbol{p}_{1}, \ldots, \boldsymbol{p}_{n}\right\}$ is also associated with the set of primitive wrenches $W=\left\{\boldsymbol{\omega}_{11}, \ldots, \boldsymbol{\omega}_{1 m}, \ldots, \boldsymbol{\omega}_{n 1}, \ldots, \boldsymbol{\omega}_{n m}\right\}$.

\section{B. Force closure test and grasp quality}

A necessary and sufficient condition for the existence of an FC grasp is that the origin $O$ of the wrench space lies strictly inside the convex hull of $W$, represented as $C H(W)$ [19]. In this work, the condition $O \in C H(W)$ is checked by verifying that $O$ and the centroid $P$ of the primitive contact wrenches in $W$ (which is always an interior point of $C H(W)$ ) lie on the same side of the hyperplane $H_{k}$ containing the facet $k$ of $C H(W), \forall k$ [14].

To quantify the goodness of a grasp, the considered grasp quality measure is the largest perturbation wrench that the grasp can resist independently of the perturbation direction [20]. This grasp quality is equivalent to the radius of the largest hypersphere centered on $O$ and fully contained in $C H(W)$, i.e. it is the distance from $O$ to the closest facet of $C H(W)$.

\section{Independent contact regions}

A general procedure to compute Independent Contact Regions (ICRS), such that if each finger is located at any point inside its corresponding $\mathrm{ICR}_{i}$ an $\mathrm{FC}$ grasp with a desired minimum grasp quality $Q_{r}$ is always obtained, was presented in a previous work [14]. ICRS are computed starting from any given FC grasp. Taking into consideration the presented assumptions, each ICR is obtained as a set of neighboring points, assuming that a contact between them also generates an FC grasp.

The procedure is conceptually illustrated in Fig. 1 with a hypothetical 2D wrench space. In this space, for each physical contact point $\boldsymbol{p}_{i}$ on the object 3 wrenches are represented: one in white representing the wrench $\omega_{i}$ produced by a unitary contact force in the direction normal to the object surface, and two in black representing the primitive contact wrenches due to friction. A hypothetical $\mathrm{CH}(W)$ is illustrated with four facets $F_{k}, k=1, \ldots, 4$, involving primitive wrenches of four contact points. Now, the $\mathrm{ICR}_{1}$ is the set of neighboring points of $\boldsymbol{p}_{1}$ such that at least one of its primitive wrenches falls into the region $S_{1}$ defined by the two hyperplanes $H_{1}^{\prime \prime}$ and $H_{2}^{\prime \prime}$, which contain the origin $O$ of the wrench space and are parallel to the facets $F_{1}$ and $F_{2}$ respectively ( $F_{1}$ and $F_{2}$ include at least one primitive wrench 


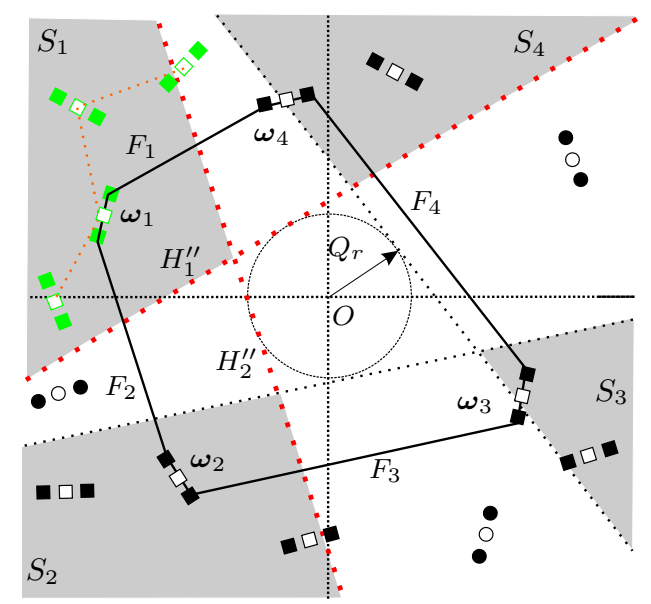

Fig. 1. Search for ICRS ensuring a minimum grasp quality. Search zones $S_{i}$ for each grasping point are depicted in gray, and the wrenches associated with neighboring points within each $\mathrm{ICR}_{i}$ are depicted with squares. The computation of $\mathrm{ICR}_{1}$ for the contact point $\boldsymbol{p}_{1}$ is illustrated.

associated with $\boldsymbol{p}_{1}$ ). Wrenches corresponding to physical points in $\mathrm{ICR}_{1}$ are represented by colored squares, and the condition of neighboring points in the physical space are represented with a link between the corresponding wrenches $\boldsymbol{\omega}_{i}$. Note that changing the contact at $\boldsymbol{p}_{1}$ on the object boundary (i.e changing the wrench $\boldsymbol{\omega}_{1}$ ) to any other point inside ICR $_{1}$ will always satisfy the FC condition with a quality $Q \geq Q_{r}$ because $C H(W)$ will always contain the origin $O$. The same principle is applied to determine the remaining $\mathrm{ICR}_{i}$, thus obtaining ICRS $=\left\{\mathrm{ICR}_{1}, \mathrm{ICR}_{2}, \mathrm{ICR}_{3}\right.$, $\left.\mathrm{ICR}_{4}\right\}$. The algorithm to compute ICRS is the following

Algorithm 1: Determination of ICRS

1) Find a starting FC grasp $C=\left\{\boldsymbol{p}_{1}, \ldots, \boldsymbol{p}_{n}\right\}$, and obtain the corresponding set $W$ and its quality $Q_{s}$

2) Select the minimum acceptable quality $Q_{r}=\alpha Q_{s}$, with $0<\alpha \leq 1$

3) Compute $C H(W)$

4) For $i=1$ to $n$ (i.e. for each contact point $\boldsymbol{p}_{i} \in C$ ), do

a) For each facet $F_{k}$ of $C H(W)$ having at least one vertex $\boldsymbol{\omega}_{i j}$, build the hyperplane $H_{k}^{\prime \prime}$ parallel to $F_{k}$ and at a distance $Q_{r}$ from the origin $O$, leaving $O$ and $F_{k}$ in different half-spaces. Let $H_{k}^{\prime \prime}$ be the open half-space such that $\boldsymbol{\omega}_{i j} \in H_{k}^{\prime \prime+}$

b) Initialize $\operatorname{ICR}_{i}=\left\{\boldsymbol{p}_{i}\right\}$

c) Label $\boldsymbol{p}_{i}$ as open

d) While there are open points $\boldsymbol{p}_{h} \in \mathrm{ICR}_{i}$, do

i) For all the neighboring points $\boldsymbol{p}_{s}$ of $\boldsymbol{p}_{h}$, do If $\exists j$ such that $\boldsymbol{\omega}_{s j} \in \bigcap_{k}{H_{k}^{\prime \prime+}}^{+}$, then

$\mathrm{ICR}_{i}=\mathrm{ICR}_{i} \cup\left\{\boldsymbol{p}_{s}\right\}$

Label $\boldsymbol{p}_{s}$ as open

ii) Label $\boldsymbol{p}_{h}$ as closed

\section{5) Return the ICRs}

Note that the sizes of the ICRS are strongly influenced by the choice of the desired minimum quality $Q_{r}$ (the larger the $Q_{r}$ the smaller the ICRS), and by the set of primitive wrenches $W$, which is directly related to the

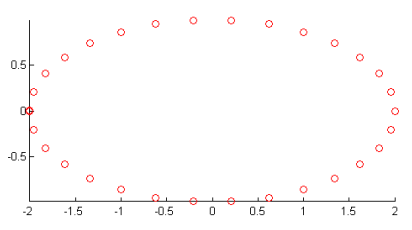

a)
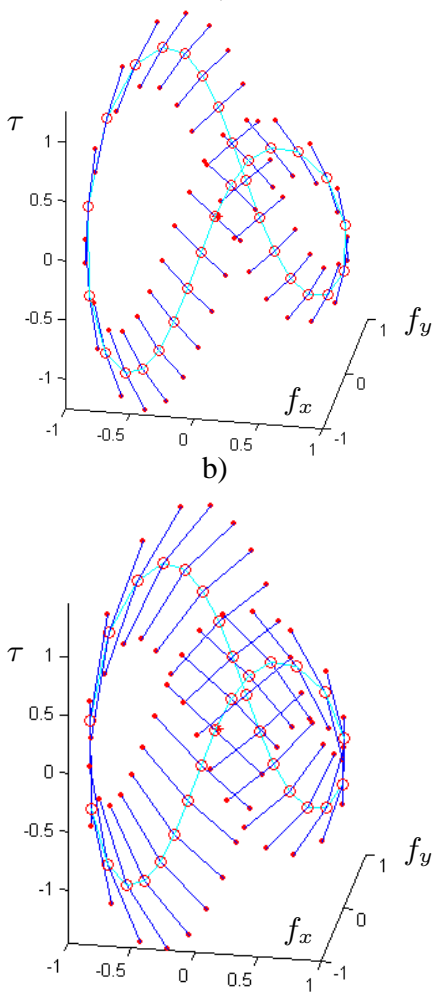

c)

Fig. 2. Representation of the friction cones in the wrench space for each contact point of a discretized ellipse: a) Discretized ellipse; b) Friction cones for $\mu=0.2$; c) Friction cones for $\mu=0.4$. Line segments join the primitive wrenches corresponding to the same friction cone.

friction coefficient (the smaller the friction coefficient, the smaller the ICRS).

\section{INFLUENCE OF FRICTION}

The real estimation of parameters that define the contact between two objects is, in general, a difficult task. On the one hand side, depending on the materials and geometry of the fingertips, a soft or hard finger contact model must be chosen to represent the actual contact between the fingertips and the object. On the other hand, the friction coefficient can be difficult to estimate from the information usually available for grasp planners. This section deals with these two factors and its influence on the computation of ICRS.

\section{A. Uncertainty in the friction coefficient}

As the material and the surface properties (e.g. roughness, deformations) for the grasped object are, in general, not well known, it is difficult to provide an exact friction coefficient between the fingers and the object. Besides, the coefficients are very sensitive to environment conditions (temperature 
or vibration, dust, oil or water on the surfaces). In general, these factors tend to diminish the nominal friction coefficient $\mu_{n o m}$. The effect of this uncertainty could be modelled as

$$
\mu_{\text {min }}=\frac{1}{\kappa} \mu_{\text {nom }}
$$

with $\kappa \geq 1$ the reduction coefficient. Fig. 2 illustrates the influence of the friction coefficient on the primitive wrenches (i.e. on the representation of a friction cone) in the wrench space for a discretized ellipse. Note that for a frictional contact point $\boldsymbol{p}_{c}$ on a $2 \mathrm{D}$ object, the possible forces applied at $\boldsymbol{p}_{c}$ within a linearized friction cone are mapped as a 2-dimensional subspace in the 3-dimensional wrench space. The points represent primitive wrenches corresponding to the friction cone at different contact points on the ellipse for a friction coefficient $\mu=0.2$ (Fig. 2b) and $\mu=0.4$ (Fig. 2c). Note that the higher the friction coefficient, the more spread are the corresponding wrenches in the wrench space, and therefore the more easier to get an FC grasp (and the larger the ICRS obtained using such FC starting grasp).

With the expression provided in (3), two different ICRS can be computed for the object:

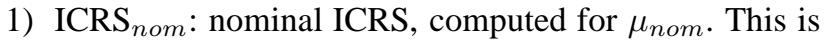
the ideal case.

2) ICRS $_{m i n}$ : minimal ICRS, computed for $\mu_{m i n}$.

Note that diminishing $\mu$ may potentially lead to a situation where the force closure property for the starting grasp cannot be guaranteed any longer. If this is the case, then the computation of ICRS using Algorithm 1 will lead to an empty set of ICRS.

The minimal ICRS (if they exist) allow an FC grasp despite any variation of $\mu$, i.e. they are the most secure ICRS to grasp the object. If at least one finger is outside its $\mathrm{ICR}_{\text {min }}$, then getting an FC grasp cannot be guaranteed due to friction uncertainty.

As an example, Fig. 3 shows the computation of the ICRS $_{n o m}$ and ICRS min $_{\text {in }}$ for a parallelepiped, with $\mu=0.2$, $\kappa=2$ and frictional cones linearized with $m=8$ sides. The real ICRS must lie in the ambiguity zone, i.e. somewhere between the ICRS nominal and minimal.

\section{B. Soft vs. hard finger}

The procedure for ICRS computation presented in Section II-C can also be extended to consider soft finger contacts by changing the set $W$ of primitive contact wrenches. The soft finger contact model assumes a finite contact area between the object and the finger, therefore allowing the application of a moment around the local surface normal. Two main models are considered in the literature for soft fingers, depending on the relation between the shear and torsional forces. For a local coordinate frame $(\boldsymbol{n}, \boldsymbol{o}, \boldsymbol{t})$ at the $i$-th contact point (with $\boldsymbol{n}$ the normal direction and $\boldsymbol{o}$ and $\boldsymbol{t}$ the tangential directions to the surface of the object), the linear (SFL) and elliptical (SFE) models state that to avoid separation and slippage at the contact point, the force $f_{i}$ must satisfy [21], [22]:

$$
S F L: 0 \leq \frac{1}{\mu} \sqrt{\boldsymbol{f}_{i o}^{2}+\boldsymbol{f}_{i t}^{2}}+\frac{1}{\mu_{l}}\left|\boldsymbol{\tau}_{i n}\right| \leq \boldsymbol{f}_{i n}
$$

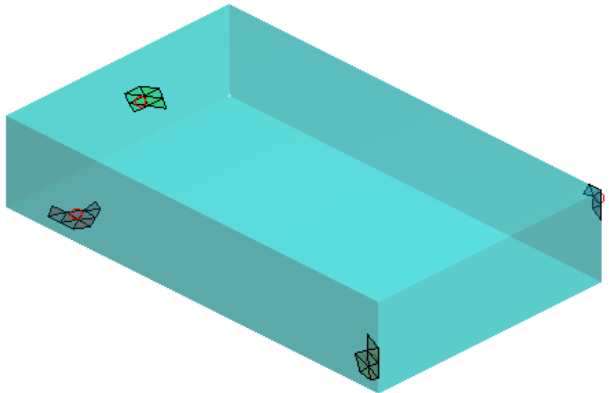

a)

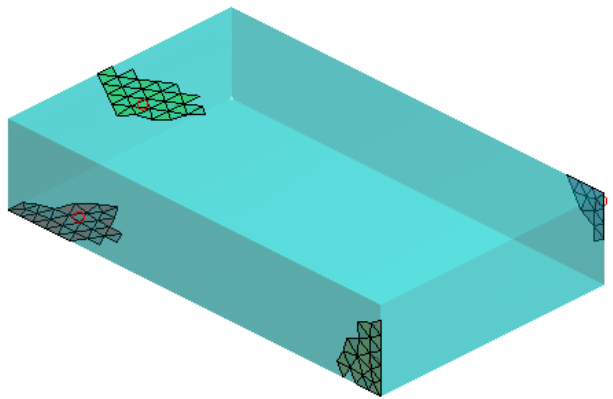

b)

Fig. 3. Independent contact regions on a parallelepiped with $Q_{r}=0.05$ : a) Minimal ICRS, $\mu_{\min }=0.1$; b) Nominal ICRS, $\mu_{\text {nom }}=0.2$. Note that the higher the friction coefficient, the larger the $\mathrm{ICR}_{i}$ obtained.

$$
S F E: 0 \leq \sqrt{\frac{1}{\mu^{2}}\left(f_{i o}^{2}+f_{i t}^{2}\right)+\frac{1}{\mu_{e}^{2}} \tau_{i n}^{2}} \leq f_{i n}
$$

with $\mu$ the coefficient of tangential friction, and $\mu_{l}$ and $\mu_{e}$ the (different) coefficients of torsional friction. To get a proper set of primitive wrenches, the SFE model can be linearized in a local coordinate frame as

$\boldsymbol{\omega}_{i}=\left[1, \mu \cos \frac{2 \pi j}{J} \cos \frac{\pi k}{2 K}, \mu \sin \frac{2 \pi j}{J} \cos \frac{\pi k}{2 K}, 0,, 0, \mu_{e} \sin \frac{\pi k}{2 K}\right]^{T}$

where $j=1$ for $k= \pm K$, and $j=1,2, \ldots, J(J \geq 3)$ for $k=-(K-1), \ldots,-1,0,1, \ldots, K-1 . J$ and $K$ are parameters that can be adjusted to improve the quality of the linearization [22].

For the SFL model, the set of primitive wrenches is linearized with

$\boldsymbol{\omega}_{i}=\left[1, \mu \cos \frac{2 \pi j}{J} \cos \frac{\pi k}{2}, \mu \sin \frac{2 \pi j}{J} \cos \frac{\pi k}{2}, 0,0, \mu_{l} \sin \frac{\pi k}{2}\right]^{T}$

where $k=-1,0,1$, with $j=1$ for $k= \pm 1$, and $j=1,2, \ldots, J(J \geq 3)$ for $k=0$. In other words, for the SFL model the set $W$ of primitive wrenches in the object coordinate frame defined in Subsection II-A simply contains two additional wrenches per finger, namely $\boldsymbol{\omega}_{i l}=\left(0_{1 \times 3} \quad \pm \hat{\boldsymbol{n}}_{i} \mu_{l}\right)^{T}$.

The consideration of a hard finger model for computing the ICRS, neglecting the torsional components of the wrench 


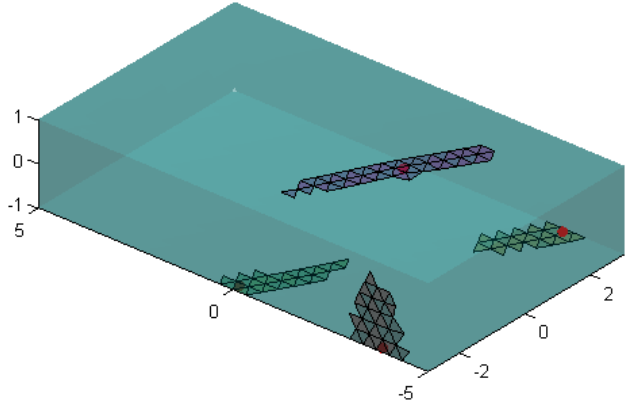

a)

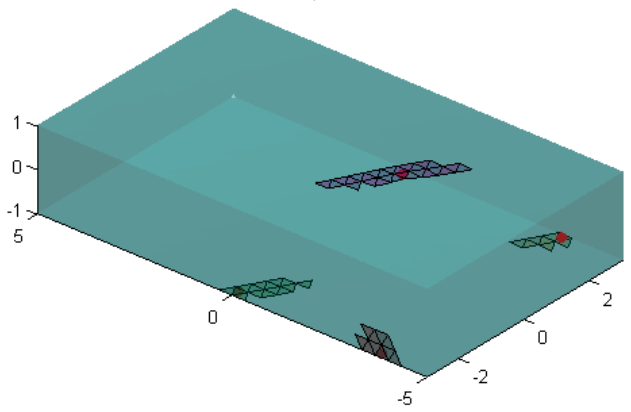

b)

Fig. 4. Independent contact regions on a parallelepiped, with minimum quality $Q_{r}=0.03$, and using: a) A soft finger model, $\mu=0.3, \mu_{l}=0.5$; b) A hard finger model, $\mu=0.3$

at the contact points, leads to smaller ICRS. To illustrate this effect, Fig. 4 shows the computation of ICRS on a parallelepiped discretized with 3422 triangles, using in both cases the same starting 4-finger FC grasp. For this object the regions computed using the soft finger model are $42 \%$ larger (in average) than the corresponding regions using the hard finger model. The relation of size between the regions computed with both models depends on the friction coefficients, the minimum quality and the starting FC grasp.

\section{UNCERTAINTIES RELATED TO THE OBJECT MODEL}

The representation of a real 3D object as a cloud of points or as a triangular mesh could involve several errors due, for instance, to possible locations occluded in the images used to build the model, or to intrinsic errors in the acquisition system. As the grasp quality depends strongly on the location of the contact points and its corresponding normal directions, the effects of geometrical uncertainties should also be considered. The goal of this section is getting a proper way to represent these uncertainties, and to include them in the computation of ICRS.

Fig. 5 shows the effect of the uncertainties in the location and normal direction at one contact point on the location of the wrench cone in the wrench space, using the same ellipse of Fig. 2a. Note the different nature of the influence of uncertainties due to position and direction, which calls for a different treatment for both sources of uncertainty.

\section{A. Uncertainty in the location of the boundary points}

The location $\boldsymbol{p}_{i b}$ of the actual boundary (contact) point is considered to be inside a closed sphere of radius $\Delta p_{i}$
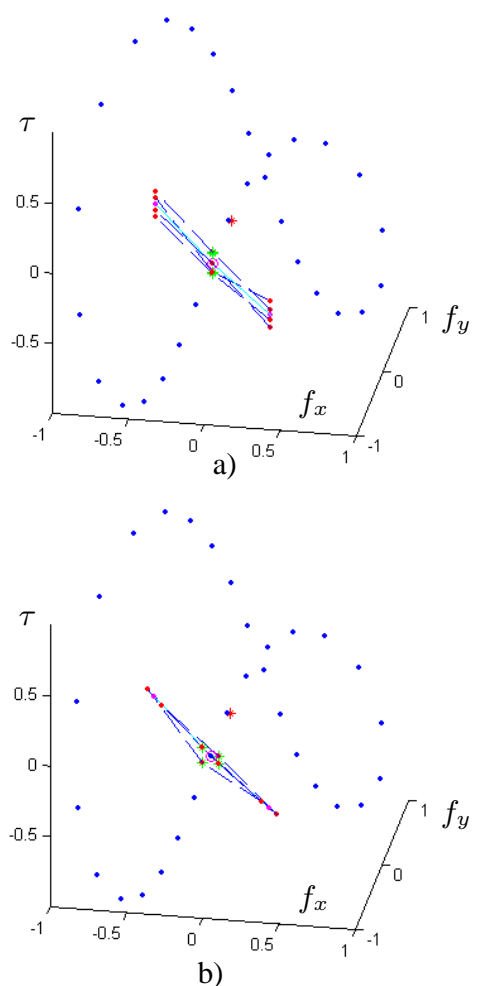

Fig. 5. Influence of uncertainties on the wrench cone for a contact on the discretized ellipse: a) Variation of $\left\|\Delta \boldsymbol{p}_{i}\right\|=0.1$ in the location of the contact point; b) Variation of $\theta=3^{\circ}$ in the normal direction.

centered at the nominal position $\boldsymbol{p}_{i}$ of the boundary point, i.e. $\boldsymbol{p}_{i b}=\boldsymbol{p}_{i}+\alpha \Delta \boldsymbol{p}_{i}$, with $0 \leq \alpha \leq 1$. The primitive wrenches produced at the potential locations of the real contact point are described with

$$
\boldsymbol{\omega}_{i j}=\left(\begin{array}{c}
\hat{\boldsymbol{n}}_{\boldsymbol{i j}} \\
\boldsymbol{p}_{i b} \times \hat{\boldsymbol{n}}_{\boldsymbol{i j}}
\end{array}\right)=\left(\begin{array}{c}
\hat{\boldsymbol{n}}_{\boldsymbol{i j}} \\
\boldsymbol{p}_{i} \times \hat{\boldsymbol{n}}_{\boldsymbol{i j}}
\end{array}\right)+\left(\begin{array}{c}
0 \\
\alpha \Delta \boldsymbol{p}_{i} \times \hat{\boldsymbol{n}}_{\boldsymbol{i j}}
\end{array}\right)
$$

Thus, the uncertainty in the location of the contact point is a perturbation $\Delta \tau$ affecting only the torque components of the wrench. Note that the magnitude of $\hat{\boldsymbol{n}}_{\boldsymbol{i} \boldsymbol{j}}$ in Eq. (8) is 1 , so the magnitude of the maximum perturbation in the torque direction is

$$
\|\Delta \boldsymbol{\tau}\|_{\max }=\left\|\Delta \boldsymbol{p}_{i} \times \hat{\boldsymbol{n}}_{\boldsymbol{i j}}\right\|=\left\|\Delta \boldsymbol{p}_{i}\right\|
$$

To illustrate the effect of this perturbation in the computation of the ICRS, Fig. 6 illustrates a hypothetical 2-dimensional wrench space, with the horizontal axis representing the force component $f$ and the vertical axis representing the torque component $\tau$ for the wrench. Let a generic hyperplane $H_{k}$ be described with the equation $\boldsymbol{e} \cdot \boldsymbol{\omega}=e_{0}$, where $e$ is the vector normal to the hyperplane. The distance of the hyperplane to the origin is given by

$$
D=\frac{\left|e_{0}\right|}{\|\boldsymbol{e}\|}
$$

Now, let every point of a hyperplane $H_{k}^{\prime \prime}$ (which partially defines a search zone $S_{i}$ in Algorithm 1, Section II-C) be moved by a distance $\Delta \tau$ in the torque direction. A new 


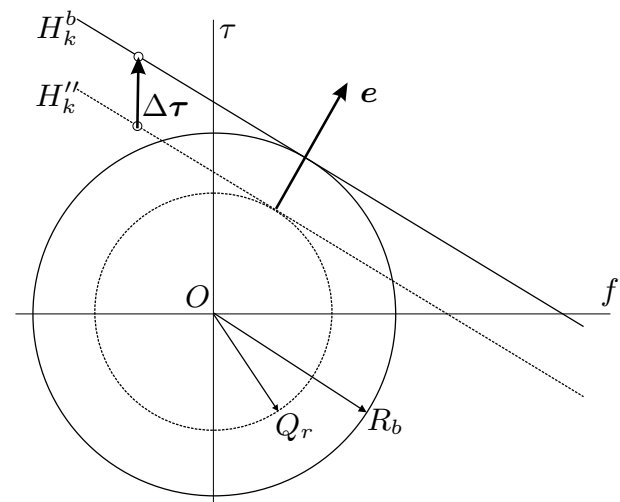

Fig. 6. Uncertainty in the contact location results in a displacement of the hyperplanes defining the search zones $S_{i}$.

hyperplane $H_{k}^{b}$ is obtained in this way, which takes into account the maximum error in the location of a contact point. The original hyperplane $H_{k}^{\prime \prime}$ is tangent to a hypersphere with radius $Q_{r}$; the new hyperplane $H_{k}^{b}$ is tangent to a hypershpere with radius $R_{b}$ given by

$$
R_{b}=Q_{r}+\Delta \tau \cdot e
$$

Note that this holds true for the 6-dimensional wrench space, as the radius $R_{b}$ is computed as the original radius plus the projection of the uncertainty $\Delta \boldsymbol{\tau}$ on the vector $\boldsymbol{e}$ normal to the hyperplane $H_{k}^{\prime \prime}$.

Therefore, the consideration of uncertainty in the location of the contact points can be taken into account with the computation of the following ICRS:

1) $\mathrm{ICRS}_{\text {nom }}$ : nominal $\mathrm{ICR}_{i}$, computed with Algorithm 1 using the nominal position $\boldsymbol{p}_{i}$ for all the contact points.

2) $\mathrm{ICRS}_{\text {min }}$ : minimal $\mathrm{ICR}_{i}$, obtained with Algorithm 1, but using in Step 4a the hyperplanes $H_{k}^{b}$ parallel to the nominal hyperplanes $H_{k}^{\prime \prime}$ with a distance to the origin given by $R_{b_{\text {min }}}=Q_{r}+\Delta \boldsymbol{\tau} \cdot \boldsymbol{e}$.

Then, the consideration of this uncertainty implies computing the ICRS with a minimum quality $R_{b}$ larger that the predefined quality $Q_{r}$. Note that the uncertainty in the location of the contact points has an upper boundary that still allows to get an FC grasp. This boundary was computed in [15]. In any case, increasing the uncertainty in position leads to smaller $\mathrm{ICR}_{i}$, eventually reaching a case where every $\mathrm{ICR}_{i}$ is empty, i.e. there is no possible FC grasp under those conditions.

\section{B. Uncertainty in the direction normal to the object bound- ary}

In order to model this uncertainty, all the potential normal directions are considered to be contained inside a cone with semiangle $\theta$ and with its axis along the nominal normal direction (Fig. 7). The real friction cone is somewhere between the minimal and maximal cones depicted in the figure. Let $\mu$ be the friction coefficient (assuming no uncertainty in its determination, or considering $\mu$ as a conservative friction coefficient). The friction cones have a semiangle of:

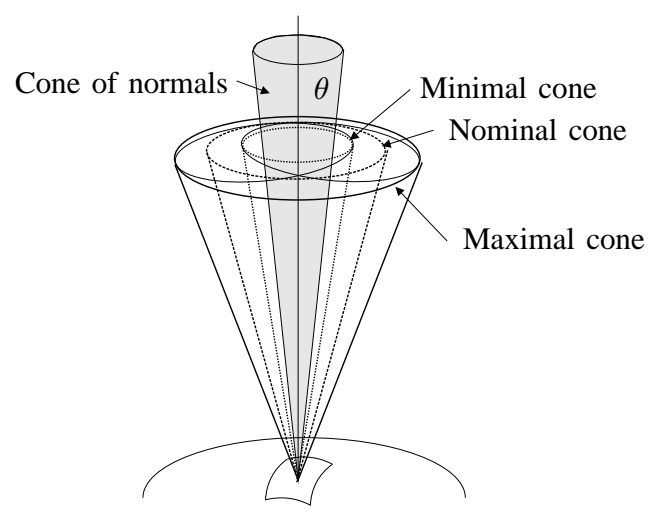

Fig. 7. Uncertainties in the normal direction define a cone of normals containing all the possible normal directions. All the potential friction cones can be found between a minimal and a maximal cone.

$$
\begin{aligned}
& \text { Minimal cone: } \theta_{\min }=\operatorname{atan}(\mu)-\theta \\
& \text { Maximal cone: } \theta_{\max }=\operatorname{atan}(\mu)+\theta
\end{aligned}
$$

Therefore, a minimal and a maximal $\mathrm{ICR}_{i}$ are computed for each point, using pseudo-friction coefficients of $\mu_{\text {min }}=\tan \left(\theta_{\min }\right)$ and $\mu_{\max }=\tan \left(\theta_{\max }\right)$, respectively. The real $\mathrm{ICR}_{i}$ is somewhere between these two boundaries; using ICRS $_{\text {min }}$ leads to a safe (conservative) consideration of this uncertainty. However, note that the FC condition can only be guaranteed if $\mu_{\min }>0$. The limit case appears when the direction uncertainty is $\theta=\operatorname{atan}(\mu)$. In that case, the contact forces may only be applied along the direction normal to the object surface, i.e. the contacts become frictionless, and therefore the FC condition cannot be guaranteed if $n<7$.

Fig. 8 shows the computation of the ICRS for a workpiece proposed in [6], discretized with 3946 triangles and using $n=4$ fingers, $\mu=0.2$, and frictional cones linearized with $m=8$ sides. It is considered that the normals to the object surface have an uncertainty of $\theta=3^{\circ}$, and $\left\|\Delta \boldsymbol{p}_{i}\right\|=0.5$ is the uncertainty in the location of the boundary points.

\section{CONCLUSIONS}

This paper has discussed the influence of uncertainties on the computation of Independent Contact Regions to grasp a discrete 3D object. First, the effect of the contact type was presented, including the linearization of the friction cone required to use a soft finger model in the computation of ICRS. The influence of the uncertainty in the friction coefficient was also discussed.

The consideration of uncertainties in the location of the contact points is equivalent to consider a change in the minimum quality used to compute the ICRS. The uncertainty in the normal direction to the object surface was tackled by reformulating the problem as the computation of ICRS using equivalent pseudo-coefficients of friction. In all the cases, a minimal Independent Contact Region is always computed, such that it ensures always an FC grasp. As expected, the existence of an FC grasp is compromised when there is significant uncertainty that may produce an empty $\mathrm{ICR}_{i}$. 


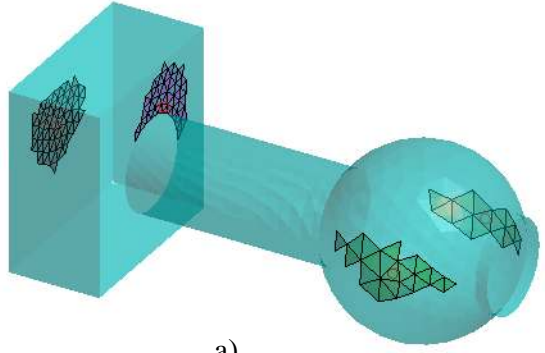

a)

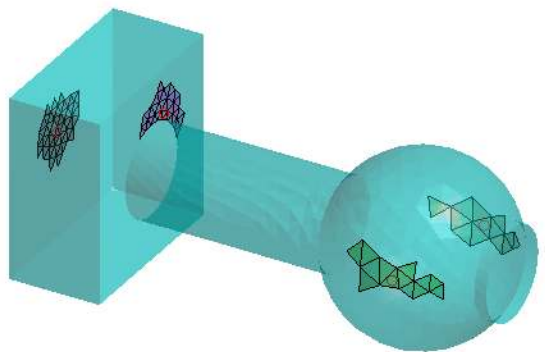

b)

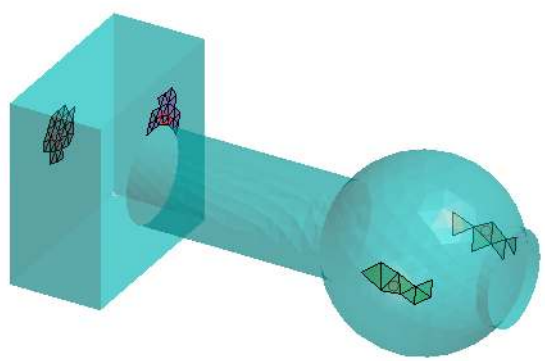

c)

Fig. 8. Independent contact regions on a workpiece: a) Nonimal ICRS (no uncertainty), $\mu_{\min }=0.3$, minimum quality $Q_{r}=0.05$; b) Minimal ICRS, $\mu_{\text {min }}=0.2438$ (considering uncertainty in the normal direction), minimum quality $Q_{r}=0.05$; c) Minimal ICRS with the combined effect of uncertainty in the normal direction and in the location of the contact points $\left(\left\|\Delta \boldsymbol{p}_{i}\right\|=0.5\right)$.

The considerations presented in this paper enhance the real applicability of ICRS, as they can now take into account uncertainties coming from the real world, such as those produced by an imperfect discrete model of a real continuous physical object.

\section{REFERENCES}

[1] A. Bicchi, "On the closure properties of robotic grasping," Int. $J$. Robotics Research, vol. 14, no. 4, pp. 319-344, 1995.
[2] X. Markenscoff, L. Ni, and C. Papadimitriou, "The geometry of grasping," Int. J. Robotics Research, vol. 9, no. 1, pp. 61-74, 1990.

[3] Y. Liu, "Computing n-finger form-closure grasps on polygonal objects," Int. J. Robotics Research, vol. 19, no. 2, pp. 149-158, 2000.

[4] N. Niparnan and A. Sudsang, "Computing all force-closure grasps of 2D objects from contact point set," in Proc. IEEE/RSJ Int. Conf. Intelligent Robots and Systems, 2006, pp. 1599-1604.

[5] J. Ponce, S. Sullivan, A. Sudsang, J. Boissonat, and J. Merlet, "On computing four-finger equilibrium and force-closure grasps of polyhedral objects," Int. J. Robotics Research, vol. 16, no. 1, pp. 1135, 1997.

[6] X. Zhu and J. Wang, "Synthesis of force-closure grasps on 3-D objects based on the Q distance," IEEE Trans. Robotics and Automation, vol. 19, no. 4, pp. 669-679, 2003.

[7] Y. Liu, M. Lam, and D. Ding, "A complete and efficient algorithm for searching 3-D form closure grasps in the discrete domain," IEEE Trans. Robotics, vol. 20, no. 5, pp. 805-816, 2004.

[8] M. Roa and R. Suárez, "Finding locally optimum force-closure grasps," J. Robotics and Computer Integrated Manufacturing, vol. 25, no. 3, pp. 536-544, 2009.

[9] V. Nguyen, "Constructing force-closure grasps," Int. J. Robotics Research, vol. 7, no. 3, pp. 3-16, 1988.

[10] C. Rosales, L. Ros, J. Porta, and R. Suarez, "Synthesizing grasp configurations with specified contact regions," Int. J. Robotics Research, DOI: 10.1177/0278364910370218, 2010.

[11] J. Cornellà and R. Suárez, "Fast and flexible determination of forceclosure independent regions to grasp polygonal objects," in Proc. IEEE Int. Conf. Robotics and Automation, 2005, pp. 778-783.

[12] — , "Determining independent grasp regions on 2D discrete objects," in Proc. IEEE/RSJ Int. Conf. Intelligent Robots and Systems, 2005, pp. 2936-2941.

[13] N. Pollard, "Closure and quality equivalence for efficient synthesis of grasps from examples," Int. J. Robotics Research, vol. 23, no. 6, pp. 595-614, 2004.

[14] M. Roa and R. Suárez, "Computation of independent contact regions for grasping 3-D objects," IEEE Trans. Robotics, vol. 25, no. 4, pp. 839-850, 2009

[15] Y. Zheng and W. Qian, "Coping with the grasping uncertainties in force-closure analysis," Int. J. Robotics Research, vol. 24, no. 4, pp. 311-327, 2004.

[16] V. Christopoulus and P. Schrater, "Handling shape and contact location uncertainty in grasping two-dimensional planar objects," in Proc. IEEE Int. Conf. Intelligent Robots and Systems, 2007, pp. 1557-1563.

[17] A. Morales, P. Sanz, and A. del Pobil, "Vision-based computation of three-finger grasps on unknown planar objects," in Proc. IEEE/RSJ Int. Conf. Intelligent Robots and Systems, 2002, pp. 1711-1716.

[18] K. Hsiao, S. Chitta, M. Ciocarlie, and E. G. Jones, "Contact-reactive grasping of objects with partial shape information," in Proc. IEEE/RSJ Int. Conf. Intelligent Robots and Systems, 2010.

[19] R. Murray, Z. Li, and S. Sastry, A Mathematical Introduction to Robotic Manipulation. Boca Raton, Florida: CRC Press, 1994.

[20] C. Ferrari and J. Canny, "Planning optimal grasps," in Proc. IEEE Int. Conf. Robotics and Automation, 1992, pp. 2290-2295.

[21] L. Han, J. Trinkle, and Z. Li, "Grasp analysis as linear matrix inequality problems," IEEE Trans. Robotics and Automation, vol. 16, no. 6, pp. 663-674, 2000.

[22] Y. Zheng and W. Qian, "Linearizing the soft finger contact constraint with application to dynamic force distribution in multifingered grasping," Science in China E: Engineering \& Materials Science, vol. 48, no. 2, pp. 121-130, 2005. 